\title{
Functional Near-Infrared Spectroscopy and Electroencephalography: A Multimodal Imaging Approach
}

\author{
Anna C. Merzagora ${ }^{1}$, Meltem Izzetoglu ${ }^{1}$, Robi Polikar ${ }^{1,2}$, Valerie Weisser ${ }^{3}$, \\ Banu Onaral $^{1}$, and Maria T. Schultheis ${ }^{1,3}$ \\ ${ }^{1}$ School of Biomedical Engineering, Science and Health Systems, Drexel University, 3141 \\ Chestnut Street, Philadelphia PA 19104, U.S.A. \\ ${ }^{2}$ Department of Electrical and Computer Engineering, Rowan University, 201 Mullica Hill \\ Road, Glassboro NJ 08028, U.S.A. \\ ${ }^{3}$ Department of Psychology, Drexel University, 3141 Chestnut Street, Philadelphia PA 19104, \\ U.S.A. \\ \{a.merzagora, meltem.alkan.izzetoglu\} @drexel.edu, \\ polikar@rowan.edu, \{vdw25, banu.onaral, schultheis\} edrexel.edu
}

\begin{abstract}
Although neuroimaging has greatly expanded our knowledge about the brain-behavior relation, combining multiple neuroimaging modalities with complementing strengths can overcome some limitations encountered when using a single modality. Valuable candidates for a multimodal approach are functional near-infrared spectroscopy (fNIRS) and electroencephalography (EEG). fNIRS is an imaging technology that localizes hemodynamic changes within the cortex. However, hemodynamic activation is an intrinsically slow process. On the other hand, EEG has excellent time resolution by directly measuring the manifestation of the brain electrical activity at the scalp. Based on their complementary strengths, the integration of fNIRS and EEG may provide higher spatiotemporal resolution than either method alone. In this effort, we integrate fNIRS and EEG to evaluate the behavioral performance of six healthy adults in a working memory task. To this end, features extracted from fNIRS and EEG were used separately, as well as in combination, and their performances were compared against each other.
\end{abstract}

Keywords: multimodal neuroimaging, functional near-infrared spectroscopy, EEG, pattern classification, working memory, n-back, P300.

\section{Introduction}

The last 20 years have seen a rapid advance in neuroimaging technologies that are now widely used for non-invasive investigation of human brain functions. Application of these technologies to the fields of basic and clinical neuroscience has greatly expanded our knowledge about brain activity associated with perceptual, cognitive, emotional and behavioral processes, in health [1],[2] and disease [3],[4]. In particular, neuroimaging techniques have contributed to the investigation of the specialization 
and integration of different cerebral areas in the normal brain and to the study of brain dysfunction in varying disorders [5],[6]. Nonetheless, the current understanding of the relation between brain activity and behavior is still limited. One of the restricting factors is the inherent complexity of the system to be investigated. In fact, most task designs in neuroimaging aim at probing or manipulating one cognitive domain at a time, but human behavior results from the interaction of multiple components (e.g., attention, orienting response, planning or short-term memory). Additionally, the macroscopic brain activity is a multifaceted process and the combined use of multiple neuroimaging technologies could capture different aspects of this process.

On one hand, techniques such as electroencephalography (EEG) and magnetoencephalography (MEG) record the integrated and synchronized electromagnetic activity of populations of pyramidal neurons in the cerebral cortex [7],[8]. Both EEG and MEG have excellent temporal resolution (at the millisecond level), but they also share the weakness of a poor three-dimensional spatial localization: the activated cortical sources need to be estimated based on the distribution of the electromagnetic fields on the scalp ("inverse problem"), which is a mathematically ill-posed problem.

On the other hand, more recent neuroimaging technologies focus on hemodynamic changes, an indirect measure of brain function. These changes consist in variations in regional blood flow, in blood oxygenation or in local metabolism and are generally assumed to reflect changes in the neural activity [9]. The neuroimaging modalities in this group are functional magnetic resonance imaging (fMRI), functional nearinfrared spectroscopy (fNIRS), positron emission tomography (PET) and single photon emission computed tomography (SPECT). In contrast to EEG and MEG, the hemodynamic-based technologies offer the advantage of providing information about the spatial location of the recorded activity (with a resolution down to few millimeters). However, hemodynamic changes are intrinsically slow processes, happening in the range of seconds [10], thus limiting the temporal resolution of the recordings.

Therefore, given the complexity of the investigated processes and the wide range of characteristics for the different imaging technologies, the use of multimodal approaches is gaining the interest of the scientific community [11],[12],[13],[14]. The underlying principle is that all neuroimaging techniques provide in vivo measures of brain function but each has its own set of assets and drawbacks. Hence, the combination of multiple imaging modalities with complementing strengths can partially overcome the limitations encountered by each individual modality.

Two valuable candidates for a multimodal approach are fNIRS and EEG. fNIRS is a brain imaging technology that relies on optical techniques to detect changes in the hemodynamic activity within the cortex in response to sensory, motor, or cognitive activation [15],[16]. fNIRS relies on the placement of near-infrared light sources and detectors on the scalp. Oxygenated ( $\mathrm{HbO} 2)$ and deoxygenated $(\mathrm{HHb})$ hemoglobin are the dominant light absorbing elements within the brain at the near infrared wavelengths and have different absorption patterns of light. Thus, fNIRS can record changes in $\mathrm{HbO} 2$ and $\mathrm{HHb}$ concentrations, which occur during brain activation [17]. Similar to other hemodynamic-based neuroimaging modalities, fNIRS is able to provide information about the specific localization of the recorded hemodynamic activity. Compared to fMRI or PET, however, fNIRS is affordable and easily implementable in a portable system, allowing for a wider range of applications. By associating fNIRS with EEG, we can additionally take advantage of the good temporal 
resolution provided by the latter. After all, EEG can capture information about rapid cortico-cortical or thalamocortical oscillations that play a crucial role in the elaboration and integration of information in cognitive networks. Therefore, based on the complementary strengths offered by EEG and fNIRS, their integration has the potential to provide higher spatio-temporal resolution than either method alone.

\section{Example of Multimodal Approach}

\subsection{Background}

We provide here an example of multimodal imaging approach using fNIRS and EEG. The aim is evaluate the performance level of subjects during a task with high working memory load. This was pursued by using measures from fNIRS or EEG individually or in a combination, the results of which were then compared. The rationale for using these modalities among others is two-fold. First, there are indications that the oxygenation changes recorded by fNIRS in working memory tasks are related to the task load and at the same time are affected by the performance level of the subject [18]. Second, EEG has been extensively applied in working memory research. In particular, many studies focused on the P300 component, a peak occurring about $300 \mathrm{~ms}$ after a target stimulus presentation and reflecting the demand on attentional resources [19]. Based on its neuropsychological interpretation, the P300 amplitude is expected to increase with increasing task complexity [20], but studies have shown a decline when the stimulus is objectively harder to discriminate or when the subject is less confident in its discrimination [21]. Hence, the combined use of fNIRS and EEG can provide insight into the different mechanisms underlying the observed low performance on a working memory task.

\subsection{Experimental Protocol}

Six subjects ( 3 males and 3 females) were selected from a larger pool of healthy participants. All subjects were right-handed, with vision correctible to 20/20. Participants denied any history of neurological disorders, psychiatric illness, substance abuse or being on any current medication. The experimental protocol was approved by the Institutional Review Board at Drexel University and all participants gave their informed consent. The mean age of the participants was 24.3 years (standard deviation $=5.5$ years).

EEG activity was recorded from $12 \mathrm{Ag} / \mathrm{AgCl}$ electrodes placed at frontal, central, parietal and occipital locations according to the International 10-20 System (F7, F3, $\mathrm{Fz}, \mathrm{F} 4, \mathrm{~F} 8, \mathrm{C} 3, \mathrm{Cz}, \mathrm{C} 4, \mathrm{P} 3, \mathrm{Pz}$, and $\mathrm{Oz}$ ). All electrodes were referenced to linked mastoid leads. Vertical and horizontal electrooculograms (VEOG and HEOG) were monitored via electrodes placed above and below the left eye, and at the left and right outer canthi, respectively. EEG signals were collected using NuAmp amplifier (Neuroscan Inc., El Paso, TX); all impedances were systematically kept below $10 \mathrm{k} \Omega$ and the amplification was set to $50 \mathrm{mV} / \mathrm{mm}$. EEG signals were filtered between 0.15 and $100 \mathrm{~Hz}$ and sampled at 500 samples/second.

The hemodynamic activity of the prefrontal cortex was recorded using a continuous-wave fNIRS device first described by Chance et al. [22] and further developed at 
Drexel University (Philadelphia, PA). The system consisted of three modules: a flexible headpiece, a control box for hardware management and a computer that runs the data acquisition. The headpiece holds four light sources and 10 photodetectors, with a source-detector separation of $2.5 \mathrm{~cm}$, providing a penetration depth of approximately $1.25 \mathrm{~cm}$. The four light sources were activated in turns: each source shone light with input intensity $I_{0}$ and the four photodetectors surrounding the currently active source measured the intensity $I$ of the emerging light. The arrangement of sources and detectors on the headpiece and the configuration for data acquisition yields a total of 16 active optodes, which were designed to image cortical areas that correspond to the dorsal and inferior frontal cortices [16]. Each source emitted light at two different wavelengths in the near-infrared spectrum, namely at 730 and $850 \mathrm{~nm}$, and measures of emerging light intensity were obtained for each optode with a sampling frequency of 2 samples/second.

Participants were seated in a dimly-lit, sound attenuated room and were asked to perform a visual $n$-back task, a task widely used to investigate working memory processes [23]. Stimuli were single consonants presented in a pseudo-random sequence on a computer screen. Stimulus duration was $500 \mathrm{~ms}$, with a 2500 -ms interstimulus interval. Four conditions were used to incrementally vary the working memory load from zero to three items. In the 0-back condition, subjects responded to a single prespecified target letter (e.g. " $X$ ") with their dominant hand (pressing a button to identify the target). In the 1-back condition, the target was defined as any letter identical to the one immediately preceding it (i.e., one trial back). In the 2-back and 3-back conditions, the targets were defined as any letter that was identical to the one presented two or three trials back, respectively. The target probability was about $33 \%$ for each condition. This strategy incrementally increased working memory load from the 0 -back to the 3-back condition. Seven blocks, each containing the four conditions ( 0 -, 1-, 2- and 3-back), were presented to the subjects. The sequence of the four conditions in the seven blocks was randomized. Each presentation of the n-back conditions was followed by a $15 \mathrm{~s}$ rest period.

\subsection{Data Processing}

Information about the behavioral performance in the task was recorded for all subjects. The percentage of correct responses was calculated separately for the four working memory loads and for the overall test. Out of the total pool of subjects, 3 were randomly selected from the group with an overall performance higher than the median ("high performing" group) and 3 were randomly selected from the group with an overall performance below the median ("low performing" group). Table 1 summarizes the behavioral performance for the overall group of subjects.

fNIRS Recordings. fNIRS data were divided into blocks locked to the repeated presentations of the four working memory conditions. Each block lasted $70 \mathrm{~s}$ and a $5 \mathrm{~s}$ rest baseline was included. The raw data about light absorption acquired by the fNIRS device were low-pass filtered and were converted to changes in concentration of $\mathrm{HbO} 2$ and $\mathrm{HHb}$ using the modified Beer-Lambert law [24]. The baseline condition used in the modified Beer-Lambert law was the rest period immediately preceding each block. For each of the seven presentations of the 3-back condition, the mean 
change in $\mathrm{HbO} 2$ concentration was extracted. In particular, the channels of most interest were those monitoring the rostral portion of the superior and middle frontal gyri in the left hemisphere, since they have been previously demonstrated to be significantly activated by the n-back task [18]. Therefore, for each subject, average $\mathrm{HbO} 2$ values for each of the seven presentations of the 3-back condition were extracted from channels 4, 5 and 6 and used as features in the subsequent classification. Only the 3-back condition was investigated, based on earlier evidence that the oxygenation values recorded during the 3-back condition are affected by the performance level [18].

EEG Recordings. Independent component analysis was used to minimize ocular artifacts in the EEG recordings [25]. Stimulus-locked event-related potentials (ERPs) were extracted from channels $\mathrm{Cz}$ and $\mathrm{Pz}$ for target stimuli presented in the 3-back condition. A $150 \mathrm{~ms}$ pre-stimulus baseline window and a $700 \mathrm{~ms}$ post-stimulus response window were used. All epochs were baseline corrected by subtracting the mean of the baseline window form the full epoch. Epochs containing significant movement or muscle artifacts were discarded. The P300 peak was automatically identified at each of the two channels as the largest positive deflection in the $250-600 \mathrm{~ms}$ post-stimulus response. For each subject, the average amplitude values of the P300 peak at $\mathrm{Cz}$ and $\mathrm{Pz}$ were obtained for each of the seven presentations of the 3-back condition and used as features in the classification.

Table 1. Statistics of the behavioral performance (\%) in the n-back task for the total pool of subjects ${ }^{1}$

\begin{tabular}{llll}
\hline & Mean & (95\% Confidence Interval) & Median \\
\hline 0-back condition & $90.07 \%$ & $(81.29-98.84 \%)$ & \\
1-back condition & $92.06 \%$ & $(85.63-98.47 \%)$ & \\
2-back condition & $85.33 \%$ & $(79.23-91.42 \%)$ & \\
3-back condition & $78.07 \%$ & $(73.76-82.37 \%)$ & \\
Overall & $86.38 \%$ & $(80.48-92.28 \%)$ & $89.70 \%$ \\
\hline
\end{tabular}

Classification. The classification between "high performing" and "low performing" subjects was performed using five different features: two features were obtained from the EEG recordings (the amplitude of the P300 peak at channels $\mathrm{Cz}$ and $\mathrm{Pz}$ ) and three were obtained from the fNRIS recordings (mean change in $\mathrm{HbO} 2$ concentration at channels 4, 5 and 6).

For each subject multiple instances of these features were extracted, one for each presentation of the 3-back condition. Each instance $\left(\boldsymbol{x}_{i}\right)$ was associated with a label $y_{i}$ that stated the group of the subject from which the instance was collected $\left(y_{i}=\right.$ "low performing" or $y_{i}=$ "high performing"). The total number of instances $\boldsymbol{x}_{i}$ was 38 (7 blocks presented to 5 subjects +3 blocks presented to 1 subject) and constituted the overall set $\boldsymbol{S}$ of available instances: $\boldsymbol{S}=\left[\boldsymbol{x}_{i}, y_{i}\right]$.

Four different approaches were evaluated to determine their ability to identify "high performing" or a "low performing" individuals:

${ }^{1}$ For one subject, in the "low performing" group, only three of the seven blocks could be presented. 
1. EEG-based classification: only the features extracted from EEG recordings were used; the feature vector consisted of two elements: the P300 amplitude at $\mathrm{Cz}$ and $\mathrm{Pz}$.

2. fNIRS-based classification: only the features extracted from fNIRS recordings were used; the feature vector consisted of three elements: the mean change in $\mathrm{HbO} 2$ concentration at channels 4,5 and 6.

3. Feature-level fusion: features extracted from both EEG and fNIRS recordings were used and combined in a single feature vector of five elements.

4. Decision-level fusion: the classification was performed separately using the EEG and fNIRS features, whose results were then combined to reach the final decision.

Two types of classifiers were investigated to be used in the above mentioned approaches: the Mahalanobis discriminant (MD) and the quadratic classifier (QC). The $\mathrm{MD}$ is equivalent to the optimum Bayes classifier if the data are normally distributed with identical (although arbitrary) covariance matrices for all classes [26]. In QC, instances are labeled using a Bayesan error minimization approach, under the more general hypothesis that the covariance matrices for all classes can assume any arbitrary value [26].

For training and testing, a modified $\mathrm{k}$-fold $(k=5)$ cross-validation was implemented. In such an approach, the set $S$ is partitioned into $k$ blocks, each one representing the two groups ("low performing" and "high performing") in an approximately balanced way. Each of the $k$ blocks was in turn held out for testing $\left(S^{(k)}\right)$, while the other $k-1$ blocks $\left(\boldsymbol{S}^{(k-1)}\right)$ were used for training using a bagging procedure [27]. In bagging, an ensemble of classifiers is created: in this study the ensemble was comprised of 10 classifiers all sharing the same architecture but trained on different randomly generated subsets $\left(\boldsymbol{T} \boldsymbol{S}_{(r)}^{(k-1)} \quad \mathrm{r}=1,2, \ldots, 10\right)$ of $\boldsymbol{S}^{(k-1)}$. A label $\hat{y}_{(r)}$ is assigned to each instance in the testing set $\boldsymbol{S}^{(k)}$ by each of the 10 classifiers, which are then combined using a majority voting decision rule. In our implementation, this entire process - of generating 10 training subsets and training 10 corresponding classifiers - was repeated 5 times, each time holding out a different subset $\boldsymbol{S}^{(k)}$ for testing. For each of these 5 repetitions, the accuracy, defined as the probability of correctly classifying an instance, was calculated.

\subsection{Results}

Table 2 summarizes the behavioral performance in the four n-back conditions for the 3 subjects in the "high performing" group and for the 3 subjects in the "low performing" group. The difference in behavioral performance between the two groups is evident in the overall percentage of correctly identified stimuli and in each of the three n-back conditions.

The distribution, in the features space, of the instances collected from the two groups of individuals is presented in Fig. 1A and Fig. 1B. These figures show the features extracted respectively from the EEG recordings (P300 amplitude at Pz and $\mathrm{Cz}$ ) and from the fNIRS recordings (change in $\mathrm{HbO} 2$ concentration at channels 4, 5 and 6); in both spaces the two classes are substantially overlapping. A separate analysis confirmed also a dissociation in the $\mathrm{HbO} 2$ values during the 3-back condition 
between the two groups: whereas for the "high performing" group the $\mathrm{HbO} 2$ values were increasing with the working memory load (1-back condition: $0.0086 \mathrm{mM}$; 2back condition: $0.0096 \mathrm{mM}$; 3-back condition: $0.0216 \mathrm{mM}$ ), for the "low performing" group this relation was lost and the 3-back condition saw a decrease in the $\mathrm{HbO} 2$ values (1-back condition: $-0.0213 \mathrm{mM}$; 2-back condition: $0.0263 \mathrm{mM}$; 3-back condition: $-0.0245 \mathrm{mM})$.

Table 3 presents the accuracy obtained by the different classification strategies using each of the two classifiers (QD and MD) as base classifiers. In general, the results showed an enhancement in classification performance when features from both EEG and fNIRS are used compared to the results obtained when using them separately. In fact, the mean accuracy for the feature-level fusion and decision-level fusion strategies were overall higher than the mean accuracy for the EEG-based and fNIRS-based classifications.

Table 2. Mean behavioral performance (\%) statistics in the n-back task for the "low performing" and "high performing" group. Behavioral performance was calculated as the percentage of presented stimuli that the subject correctly identified as targets or non-targets.

\begin{tabular}{lll}
\hline & Low performing & High performing \\
\hline 0-back condition & $74.95 \%$ & $94.87 \%$ \\
1-back condition & $80.83 \%$ & $95.22 \%$ \\
2-back condition & $77.87 \%$ & $88.38 \%$ \\
3-back condition & $70.56 \%$ & $82.03 \%$ \\
Overall & $76.05 \%$ & $90.12 \%$ \\
\hline
\end{tabular}

Table 3. Mean and standard deviation of the accuracy (\%) reached by the quadratic classifier (QC) and Mahalanobis discriminant (MD) used in the four possible classification approaches. Accuracy was evaluated across the 5 repetitions of the k-fold cross-validation procedure.

\begin{tabular}{lll}
\hline & QC & MD \\
\hline EEG-based classification & $60.31 \pm 18.27 \%$ & $62.53 \pm 16.44 \%$ \\
fNIRS-based classification & $62.53 \pm 21.78 \%$ & $51.74 \pm 7.41 \%$ \\
Feature-level fusion & $65.39 \pm 16.51 \%$ & $58.09 \pm 18.56 \%$ \\
Decision-level fusion & $71.11 \pm 10.67 \%$ & $71.11 \pm 10.67 \%$ \\
\hline
\end{tabular}

Additionally, the two fusion approaches (and in particular the decision-level fusion, in agreement with [28]) provided an increase, albeit small, in the generalizing ability of the classifiers, as measured by a decrement in the accuracy standard deviation.

\subsection{Discussions}

Overall, the classification of instances collected from "high performing" and "low performing" subjects benefited from the used of combined fNIRS and EEG features. We acknowledge that the results of our statistical analyses cannot be considered conclusive at this time due to the limited data of 3 subjects (from each class) that were available to us. A larger pool of subjects, and therefore a higher number of instances available for training and testing, would allow a better estimation of the accuracy 
A

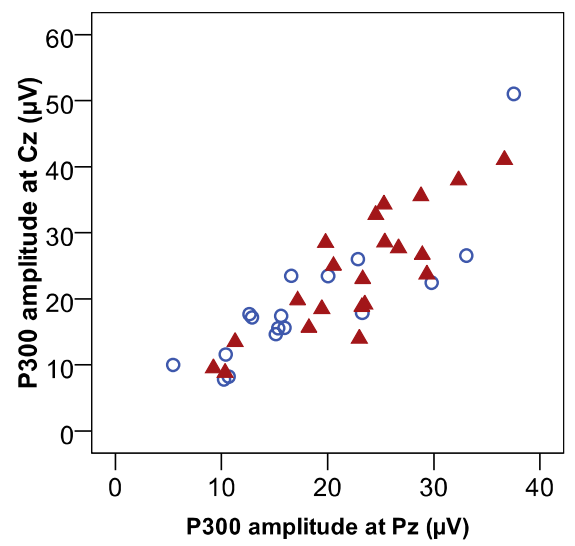

B

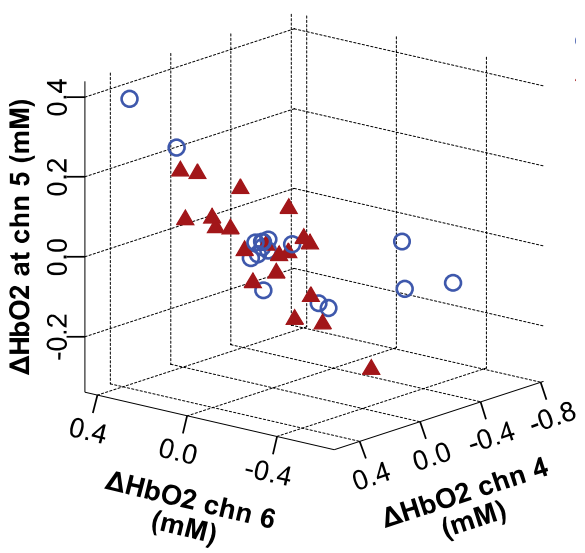

- Low performing

$\Delta$ High performing

Fig. 1. A. Scatterplot of instances of EEG-based features, the $P 300$ amplitude at $P z$ and the $P 300$ amplitude at $C z$. B. Scatterplot of instances of fNIRS-based features, the mean changes in $\mathrm{HbO} 2$ concentration at channels 4, 5 and 6 ( $\triangle \mathrm{HbO} 2$ chn4, $\triangle \mathrm{HbO} 2$ chn5 and $\triangle \mathrm{HbO} 2$ chn6, respectively ). In both part $\mathrm{A}$ and part $\mathrm{B}$, instances are categorized as belonging to high performing (filled triangles) or low performing (empty circles) individuals. From each individual, multiple instances were collected, one for each presentation of the 3-back condition.

index. Nonetheless, the combination of fNIRS and EEG improved classification accuracy, even if two relatively simple classifiers where used: a linear parametric classifier (MC) and a nonlinear parametric classifier (QC). It is reasonable to expect that higher accuracies can be obtained using more sophisticated nonlinear classifiers (such as neural networks or support vector machines), that are not bounded by assumptions about the features distributions. Similarly, the relative performance of other fusion algorithms could be investigated, ranging from the simple majority voting (presented in this paper) to multinomial methods, to the fusion of discriminant scores.

\section{Concluding Remarks}

We have investigated the feasibility and performance of fNIRS and EEG data fusion for the evaluation of the behavioral performance of six healthy adults in a working memory task. Although fNIRS and EEG have been co-registered in previous studies [29], [30], this is the first attempt at their integration by using them together in a pattern recognition application. For this study, the fNIRS-EEG fusion took advantage of both the spatial information about the hemodynamic activity (by including only the channels monitoring the rostral portion of the superior and middle frontal gyri in the left hemisphere) and the fast temporal dynamic of the cognitive processes of interest (by including information about the P300 amplitude). A similar approach can also help explain the mechanisms underlying low task performance in case of neurological 
disorders such as traumatic brain injury or multiple sclerosis, hence providing some physiological evidence important for the choice of a proper neurorehabilitation and pharmacological intervention. fNIRS-EEG fusion may further be applied to the study of other cognitive domains, in particular taking advantage of the flexibility in task designs allowed by fNIRS and EEG.

\section{References}

1. Sergent, J.: Brain-imaging studies of cognitive functions. Trends Neurosci. 17, 221-227 (1994)

2. Stufflebeam, S.M., Rosen, B.R.: Mapping cognitive function. Neuroimaging Clin N Am. 17, 469-484, viii-ix (2007)

3. Malhi, G.S., Lagopoulos, J.: Making sense of neuroimaging in psychiatry. Acta Psychiatr Scand 117, 100-117 (2008)

4. Eliassen, J.C., Boespflug, E.L., Lamy, M., Allendorfer, J., Chu, W.J., Szaflarski, J.P.: Brain-mapping techniques for evaluating poststroke recovery and rehabilitation: a review. Top Stroke Rehabil 15, 427-450 (2008)

5. Calvert, G.A.: Crossmodal processing in the human brain: insights from functional neuroimaging studies. Cereb Cortex 11, 1110-1123 (2001)

6. Lalanne, C., Lorenceau, J.: Crossmodal integration for perception and action. J. Physiol. Paris 98, 265-279 (2004)

7. Lewine, J.D., Orrison, W.W.J.: Clinical electroencephalography and event-related potentials. In: Orrison, W.W.J., Lewine, J.D., Sanders, J.A., Hartshorne, M.F. (eds.) Functional brain imaging, pp. 327-368. Mosby-Year Book, Boston (1995)

8. Lewine, J.D., Orrison, W.W.J.: Magnetoenecephalography and magnetic source imaging. In: Orrison, W.W.J., Lewine, J.D., Sanders, J.A., Hartshorne, M.F. (eds.) Functional brain imaging, pp. 369-418. Mosby-Year Book, Boston (1995)

9. Raichle, M.E., Mintun, M.A.: Brain work and brain imaging. Ann. Rev. Neurosci. 29, 449-476 (2006)

10. Logothetis, N.K., Wandell, B.A.: Interpreting the BOLD Signal. Annu. Rev. Physiol. 66, 735-769 (2004)

11. George, J.S., Aine, C.J., Mosher, J.C., Schmidt, D.M., Ranken, D.M., Schlitt, H.A., Wood, C.C., Lewine, J.D., Sanders, J.A., Belliveau, J.W.: Mapping function in the human brain with magnetoencephalography, anatomical magnetic resonance imaging, and functional magnetic resonance imaging. J. Clin. Neurophysiol. 12, 406-431 (1995)

12. He, B., Lian, J.: High-resolution spatio-temporal functional neuroimaging of brain activity. Crit. Rev. Biomed. Eng. 30, 283-306 (2002)

13. Ritter, P., Villringer, A.: Simultaneous EEG-fMRI. Neurosci. Biobehav. Rev. 30, 823-838 (2006)

14. Schiff, N.D.: Multimodal neuroimaging approaches to disorders of consciousness. J. Head Trauma Rehabil. 21, 388-397 (2006)

15. Villringer, A.: Functional neuroimaging. Optical approaches Adv. Exp. Med. Biol. 413, 118 (1997)

16. Izzetoglu, M., Izzetoglu, K., Bunce, S., Ayaz, H., Devaraj, A., Onaral, B., Pourrezaei, K.: Functional near-infrared neuroimaging. IEEE Trans Neural Syst. Rehabil. Eng. 13, 153 159 (2005)

17. Jobsis, F.F.: Noninvasive, infrared monitoring of cerebral and myocardial oxygen sufficiency and circulatory parameters. Science 198, 1264-1267 (1977) 
18. Izzetoglu, M., Bunce, S.C., Izzetoglu, K., Onaral, B., Pourrezaei, K.: Functional brain imaging using near-infrared technology. IEEE Eng. Med. Biol. Mag. 26, 38-46 (2007)

19. Kok, A.: Event-related-potential (ERP) reflections of mental resources: a review and synthesis. Biol. Psychol. 45, 19-56 (1997)

20. Johnson Jr., R.: On the neural generators of the P300 component of the event-related potential. Psychophysiology 30, 90-97 (1993)

21. Kok, A.: On the utility of $\mathrm{P} 3$ amplitude as a measure of processing capacity. Psychophysiology 38, 557-577 (2001)

22. Chance, B., Anday, E., Nioka, S., Zhou, S., Hong, L., Worden, K., Li, C., Murray, T., Ovetsky, Y., Pidikiti, D., Thomas, R.: A novel method for fast imaging of brain function, non-invasively, with light. Opt. Express 2, 411-423 (1998)

23. Owen, A.M., McMillan, K.M., Laird, A.R., Bullmore, E.: N-back working memory paradigm: a meta-analysis of normative functional neuroimaging studies. Hum. Brain Mapp. 25, 46-59 (2005)

24. Delpy, D.T., Cope, M., van der Zee, P., Arridge, S., Wray, S., Wyatt, J.: Estimation of optical pathlength through tissue from direct time of flight measurement. Phys. Med. Biol. 33, 1433-1442 (1988)

25. Jung, T.P., Makeig, S., Humphries, C., Lee, T.W., McKeown, M.J., Iragui, V., Sejnowski, T.J.: Removing electroencephalographic artifacts by blind source separation. Psychophysiology 37, 163-178 (2000)

26. Duda, R.O., Hart, P.E., Stork, D.G.: Pattern classification. Wiley Interscience, New York (2001)

27. Kuncheva, L.I.: Combining pattern classifiers: Methods and algorithms. Wiley Interscience, New York (2004)

28. Parikh, D., Polikar, R.: An Ensemble-Based Incremental Learning Approach to Data Fusion. IEEE Trans Sys. Man Cyber 37, 437-450 (2007)

29. Kennan, R.P., Horovitz, S.G., Maki, A., Yamashita, Y., Koizumi, H., Gore, J.C.: Simultaneous recording of event-related auditory oddball response using transcranial near infrared optical topography and surface EEG. Neuroimage 16, 587-592 (2002)

30. Butti, M., Pastori, A., Merzagora, A., Zucca, C., Bianchi, A., Reni, G., Cerutti, S.: Multimodal analysis of a sustained attention protocol: Continuous Performance Test assessed with Near Infrared Spectroscopy and EEG. In: 28th Annual International Conference of the IEEE EMBS, pp. 1040-1043 (2006) 